\title{
Visual movement discrimination ${ }^{1}$
}

Data are presented which support a mathematical model for visual movement perception. An observer's ability to judge the relative position of two successively presented points of light in an otherwise dark room is shown to diminish as the inter-stimulus interval is increased. However, a measure of visual position memory appears to remain invariant as this interval is varied. Neither the degree of head stabilization nor whether viewing is monocular or binocular appreciably alters this measure.

A model has been proposed by Kinchla and Smyzer (1967) for the manner in which an O compares two stimuli presented at different points in time. Of interest here is the application of the model to a visual position discrimination task in which an $O$, seated in a dark room, attempts to discriminate a lateral difference in position between two successively presented points of light. The model accounts for $O^{\prime} s$ progressive loss of accuracy as the delay between successive presentations of the lights is increased. The loss of position information during the interstimulus interval is represented in the model as a mathematical random walk process. It is further suggested that this theoretical random walk may to a large extent reflect actual involuntary eye movements during the interstimulus interval. A similar conclusion concerning the role of involuntary eye movements was arrived at by Matin, Pearce, Matin, and Kibler (1966) on the basis of direct measurement of eye movements in a situation similar to that employed by Kinchla and Smyzer. Matin et al had the $O$ fixate on a point of light for $4 \mathrm{sec}$; then, after $3 \mathrm{sec}$ in the dark, a second point of light was presented, and the $O$ judged whether it was to the left, to the right, or in the same position as the fixation light. They concluded that the $O$ appeared to be unaware of the degree to which his eye drifted away from the fixation point during the interstimulus interval and simply based his judgments on the relative points of retinal stimulation. While Matin et al derive their measure of the drift process from direct measurement of eye movements, the model proposed by Kinchla and Smyzer may allow a measure of eye drift to be derived from purely psychophysical data.

The experiment presented here provides a test of the psychophysical model based on far more data than originally reported by Kinchla and Smyzer, and it also deals with the role of head stabilization and monocular vs binocular viewing as factor in determining the $O$ 's accuracy. The $O s$ in the original Kinchla-Smyzer study sat in conventional office chairs and had no special restraints on head movement, while Matin et al employed a biting block. Since involuntary head movements could alter the point of retinal stimulation in the same manner as involuntary eye movements, the importance of head stabilization is of some interest. Furthermore, the Kinchla-Smyzer Os viewed binocularly, while Matin et al employed monocular viewing. Since the involuntary movements of each eye appear to be uncorrelated (Krauskopf, Cornsweet, \& Riggs, 1960), the relative points of retinal stimulation of each eye would not necessarily be identical in the task we have considered. If involuntary eye movements can be considered a sort of visual noise, a binocular o might combine the information from each eye to improve his performance in the same manner as noise is averaged out by combining several statistical samples.

\section{Model}

Only the essential features of the model will be discussed here since the reader may refer to the earlier paper by Kinchla and Smyzer (1967) for a more detailed development. The model consists of three processes: input, memory, and decision. The O compares a stimulus value presented at time zero, $s_{o}$, with one presented at time $t, s_{t}$, by storing the sensory input produced by $s_{o}$ in memory until st is presented. He reports a stimulus difference only if the discrepancy, denoted $y_{t}$, between the second sensory input and his memory of the first input exceeds some decision criterion, denoted $C_{t}$. In the simplest form of the model the sensory input is identical to the stimulus value, and the only information loss is in the memory where the original input is modified through a random walk process until time $t$. This produces an essentially Gaussian distribution of discrepancies, $y_{t}$ values, whose expected value is the actual stimulus difference $\left(s_{t}-s_{o}\right)$ and whose variance equals $\sigma_{t}^{2}$ where

$$
\sigma_{t}^{2}=\phi t
$$

Thus $\phi$, termed the diffusion rate, is simply the rate at which the variance of the theoretical distribution of discrepancies increases during the interstimulus interval.

The O's decision problem is shown graphically in Fig. 1. Two pairs of overlapping distributions of $y_{t}$ are presented, one pair for an interstimulus interval of $t$, another pair for a longer interval, $t$. In both cases the distribution on the left corresponds 

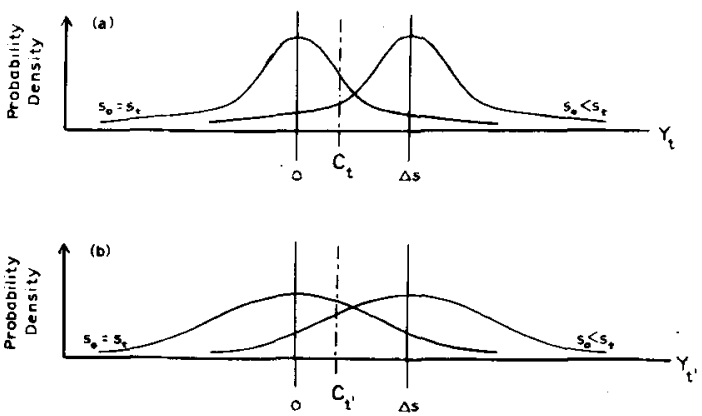

Fig. 1. Theoretical decision problem for an interstimulus interval $t$ and a longer interval $t^{*}$.

to an equal stimulus condition $\left(s_{t}=s_{0}\right)$, while the other corresponds to an unequal stimulus condition $\left(s_{t}=s_{0}+\Delta s\right)$. Note that the area to the right of the criterion value, $C_{t}$, under each curve indicates the probability of a "different" response given a particular stimulus condition. We shall term a correct report of a stimulus difference as a "hit," $\mathrm{H}$, and an incorrect report of a difference as a "false alarm," FA.

Thus the areas to the right of $C_{t}$ under the curves in Fig. 1 indicate the hit and false-alarm probabilities, denoted, respectively, $P(H)$ and $P(F A)$, for two interstimulus intervals, $t$ and $t$ '.

Note that the greater the overlap between each pair of distributions, the greater will be the P(FA) for any particular $P(H)$ regardless of the value of $C_{t}$. The degree of overlap is indicated by the measure $\delta_{t}$ where

$$
\delta_{t}=\frac{\Delta s}{\sigma_{t}}
$$

This is the familiar sensitivity measure in the Theory of Signal Detection (see Swets, 1967); i.e., the difference between the two means is expressed in units of the standard error. However, since $\sigma_{t}$ is a function of $t$ (Eq. (1)), $\delta_{t}$ is reduced as the interstimulus delay is increased; this feature is indicated in Fig. 1 by the greater overlap between the distributions for interval $t^{\prime}$.

\section{Method}

The stimulus display consisted of two circular white lights laterally separated from each other, with a luminance of $4 \mathrm{~mL}$ each. The display was placed $3.65 \mathrm{~m}$ in front of the seated $\mathrm{O}$ at eye level. The distance between the two lights subtended a visual angle of $.44^{\circ}$, and the diameter of each light subtended $.036^{\circ}$.

Each of four Os with normal visual acuity sat in complete darkness and tried to detect a lateral difference in position between two points of light presented successively with $t$ seconds between flashes. Each trial began with a 1 sec, 1000 cps auditory warning signal followed immediately by a $100 \mathrm{msec}$ duration illumination of the light on the left of the display. Then, after some time delay, either the same light or the light on the right of the display came on for another $100 \mathrm{msec}$. Following this, the $O$ had $2 \mathrm{sec}$ to indicate, by pressing an appropriate pushbutton, either that the two flashes had occurred in the same position or that the second flash was displaced to the right of the first.

The sequence of stimulus presentations was separately determined in blocks of 50 trials. The second stimulus occurred in the same position as the first on a randomly determined 25 of the 50 trials and to the right on the remaining 25 trials. Each experimental session consisted of eight 50 trial blocks with a preliminary dark adaptation period of $10 \mathrm{~min}$ and 1 min rest periods (in the dark) between blocks. There were four values of the interstimulus interval: $t$ equal to .5, 1.0, 1.5, and $2.0 \mathrm{sec}$. Each of the eight possible combinations of $t$ value with and without the biting block was in effect throughout one of the eight blocks of 50 trials. The sequence of conditions was randomly determined within each such session. During alternate, sessions the Os viewed the display either monocularly or binocularly. In all, 12 blocks of 50 trials were collected for each of the 16 combinations of four viewing conditions and four $t$ values for a total of 600 trials under each combination.

\section{Results and Discussion}

A Chi-square analysis indicated that $t$ had a statistically significant $(p<.001)$ effect on each $O$ 's performance, but that neither of the viewing variables had a statistically significant effect ( $>>.05)$.

Since the null hypothesis could not be rejected with regard to the viewing variables, the theoretical analysis of each O's data is based on the hit and false-alarm proportions at each delay, combining data from the four viewing conditions. Table 1 pre-

Table 1. Proportions of "different" responses when $s_{0}$ and $s_{t}$ actually differed, $P(H)$, when they were same, $P(F A)$, overall, $P(D)$, and the proportion of correct responses, $P(C)$, for each observer at each $t$ value.

\begin{tabular}{|c|c|c|c|c|c|c|c|c|c|c|c|c|c|c|c|c|}
\hline \multirow[b]{2}{*}{.5} & \multirow{2}{*}{$\frac{P(H)}{.84}$} & \multicolumn{2}{|c|}{$\begin{array}{l}\text { Observer } \\
\text { (FA) P(D) }\end{array}$} & \multirow{2}{*}{$\frac{1}{P(C)}$} & \multirow{2}{*}{$\frac{P(H)}{.80}$} & \multicolumn{2}{|c|}{$\begin{array}{l}\text { Observer } \\
P(F A) P(D)\end{array}$} & \multirow{2}{*}{$\frac{2}{P(C)}$} & \multicolumn{4}{|c|}{ Observer 3} & \multicolumn{4}{|c|}{ Observer 4} \\
\hline & & .22 & .53 & & & .17 & .49 & & .74 & .19 & .47 & .78 & .73 & .19 & .46 & .77 \\
\hline 1.0 & .74 & .25 & .50 & .75 & .70 & .26 & .48 & .72 & .65 & .23 & .44 & .71 & .63 & .22 & .43 & .71 \\
\hline 1.5 & .69 & .28 & .49 & .71 & .62 & .29 & .46 & .67 & .62 & .28 & .45 & .67 & .60 & .28 & .44 & .66 \\
\hline 2.0 & .70 & .33 & .52 & .69 & .62 & .30 & .46 & .66 & .57 & .29 & .43 & .64 & .60 & .30 & .45 & .65 \\
\hline
\end{tabular}


Table 2. Values of $\hat{\delta}_{\mathrm{t}}$, Pred. $\delta_{\mathrm{t}}$, and $\hat{\mathbf{c}}_{\mathrm{t}}$ from each $\mathrm{t}$ value for each observer.

\begin{tabular}{|c|c|c|c|c|c|c|c|c|c|c|c|c|}
\hline \multirow[b]{2}{*}{$\uparrow$} & \multicolumn{3}{|c|}{ Observer 1} & \multicolumn{3}{|c|}{ Observer 2} & \multicolumn{3}{|c|}{ Observer 3} & \multicolumn{3}{|c|}{$\begin{array}{r}\text { Observer } 4 \\
\hat{\phi}=.167^{*}\end{array}$} \\
\hline & $\hat{\delta}_{t}$ & Pred. $\delta_{t}$ & $\hat{C}_{t}$ & $\hat{\delta}_{+}$ & Pred. $\delta_{t}$ & $\hat{C}_{t}$ & $\hat{\delta}_{t}$ & Pred. $\delta_{t}$ & $\hat{C}_{\dagger}$ & $\hat{\delta}_{+}$ & Pred. $\delta_{t}$ & $\hat{C}_{t}$ \\
\hline .5 & 1.76 & 1.90 & .19 & 1.79 & 1.59 & .23 & 1.52 & 1.50 & .25 & 1.49 & 1.52 & .26 \\
\hline 1.0 & 1.32 & 1.34 & .22 & 1. 16 & 1.13 & .24 & 1.12 & 1.06 & .29 & 1.10 & 1.08 & .31 \\
\hline 1.5 & 1.08 & 1.09 & .24 & .86 & .92 & .27 & .88 & .87 & .29 & .84 & .88 & .30 \\
\hline 2.0 & .96 & .95 & .20 & .83 & .80 & .28 & .74 & .75 & .33 & .78 & .76 & .30 \\
\hline
\end{tabular}

* Expressed in square degrees visual angle per sec.

sents these hit and false-alarm proportions for each $O$, along with the total proportion of correct responses, $P(C)$, and the total proportion of "different" responses, $P(D)$.

While the proportion of correct responses decreased with the length of the interstimulus interval, the $O$ maintained an approximately constant proportion of "different" responses. This proportion was very nearly .5 in each case, which is consistent with the $O$ 's a priori knowledge of the stimulus distribution.

A table of normal deviates was consulted to obtain an estimate of $\delta_{t}$, denoted $\hat{\delta}_{t}$, based on each pair of hit and false-alarm proportions (this clearly follows from the definition of $\hat{\delta}_{t}$ and consideration of Fig. 1). These $\hat{\delta}_{t}$ values can be considered the observed value of $\delta_{t}$ for each $t$ value. A value of
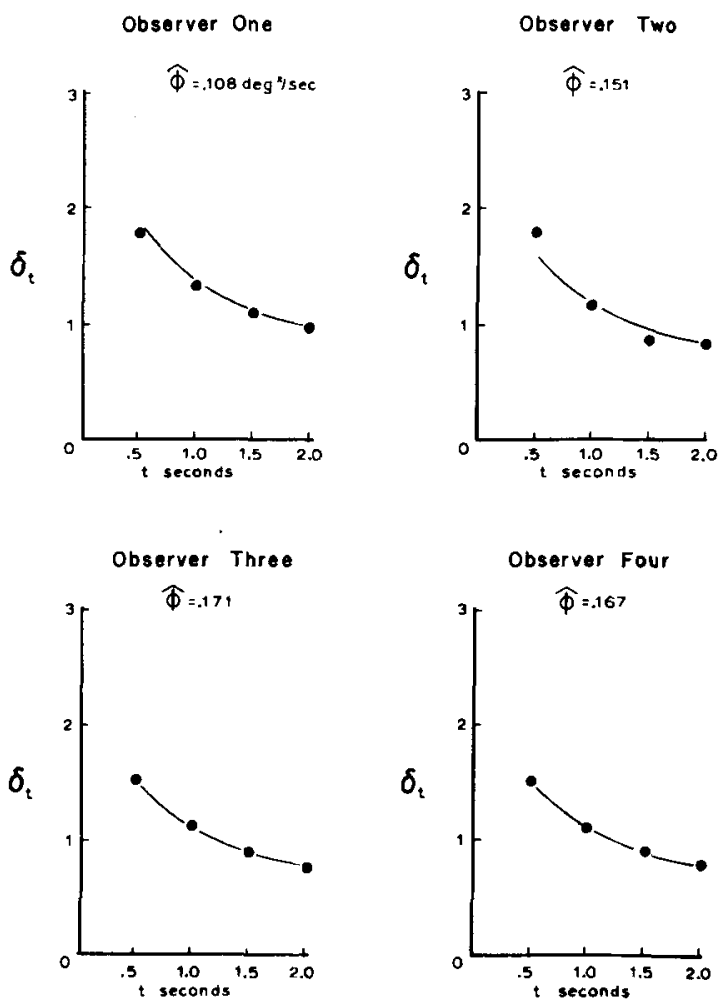

Fig. 2. Theoretical (solid lines) and observed (points) values of $\delta_{t}$ given particular values of $\phi$. $\phi$ was then selected which provided the best fit, in a least-squares sense, between these observed values of $\delta_{t}$ and predicted values of $\delta_{t}$ based on Eq. (1) and (2). These estimates of $\phi(\hat{\phi})$, the predicted $\delta_{t}$ values (Pred. $\delta_{t}$ ), and the observed $\delta_{t}$ $\left(\hat{\delta}_{t}\right)$ values are presented numerically in Table 2 and graphically in Fig. 2. A goodness-of-fit test indicated that the model accounted, on the average, for about .96 of the variance in the observed $\delta_{t}$ values. The actual proportions for Observers 1-4 were, respectively, .94, .92, .99, and .99. Thus, the model accounts for a substantial part of the total variance in $\hat{\delta}_{t}$.

Here, as in the earlier study by Kinchla and Smyzer (1967), the diffusion rates were approximately 15 square degrees per second. This is about two to three times the magnitude of variability indicated by direct measurement of involuntary eye movements 1 in the experimental situation described by Matin et al (1966). Whether this reflects an actual discrepancy between the psychophysically derived measure, $\phi$, and a measure based on direct physical measurement is not clear. Preliminary results of experiments currently being conducted in this laboratory suggest that the longer preliminary fixation exposure employed in the Matin et al situation (4 sec as compared to $.1 \mathrm{sec}$ in the present experiment) may lead to lower estimates of $\phi$. In any case, given the intermal consistency in the data as regards the influence of $t$, is seems clear that the viewing condition effects, if any, are relatively small.

Finally, it is of interest to consider estimates of the $O^{\prime} s$ decision criterion, $C_{t}$. These estimates, denoted $\hat{C}_{t}$, are simply the criterion values, given the predicted value of $\delta t$, most likely to have produced the observed hit and false-alarm proportions. These estimates are presented in Table 2. In general, as $t$ increased $C_{t}$ would have to move toward a value midway between the means of the two overlapping distributions (the point .220) in order to maintain a fixed proportion of "different" responses. Thus, the generally positive trend in $\hat{C}_{t}$ seems consistent with the apparent stability of $P(D)$ seen in Table 1.

\section{References}

Green, D. M., \& Swets, J. A. Signal detection theory and psycho- 
physics. New York: Wiley, 1966.

Kinchla, R. A., \& Smyzer, F. A diffusion model of perceptual memory. Percept. \& Psychophys., 1967, 2, 219-229.

Krauskopf, J., Comsweet, T. N., \& Riggs, L. A. Analysis of eye movements during monocular and binocular fixation. J. Opt. Soc. Amer., 1960, 50, 572-578.

Matin, L., Pearce, D., Matin, E., \& Kibler, G. Visual perception of direction in the dark: roles of local sign, eye movements, and ocular proprioception. Vis. Res., $1966,6,453-469$

\section{Note}

1. Matin, L., Columbia University, personal communication.

(Accepted for publication Novem ber 4, 1967.), 